\title{
There is Not One But Many AI: A Network Perspective on Regional Demand in AI Skills
}

\author{
(Extended Abstract - 28/02/2020)
}

\author{
Fabian Stephany ${ }^{12}$ \\ 1 Oxford Internet Institute, 1 St Giles', Oxford OX1 3JS \\ 2 Humboldt Institute for Internet and Society, Berlin, Germany \\ OrcidID: 0000-0002-0713-6010, fabian.stephany@oii.ox.ac.uk
}

\section{Introduction}

Digital technologies have a pervasive effect on our society. They augment or transform various previously analogue processes of value creation, capture, and exchange [12]. Hence, on the labour market, the skilful development and application of relevant digital technologies are in strong demand. However, early research findings indicate that the labour demand of certain tech industries is not met. The talent pool does not grow at the pace of industry demand and precise skill requirements related to growing technologies, such as Artificial Intelligence (AI), remain opaque [3]. At the same time, the mastering of AI skills becomes paramount for humans to keep a competitive edge versus increasingly independent digital technologies [10].

This work proposes a network perspective in order to empirically identify the relevant ICT skills related to AI, to what extent they are systemically related, and how their composition varies across regions. With the example of 5,227 job openings from Germany advertised as postings in Artificial Intelligence, relevant skills are identified and connected in a network fashion. Two skills are connected, if they are jointly required by the same job advertisement. Similarly, regional skill networks can be constructed: Job postings are screened by city location and skill networks are constructed for this set of regional postings exclusively. The resulting networks depict the regional city ecosystem of AI skills currently in demand.

The insights of this work can support businesses in developing a data-driven strategy for the acquisition and the development of adequate skills needed to implement and leverage new technologies at best. Furthermore, the empirical relationship of digital skill sets will help to establish a common taxonomy to be used by policy makers, education providers, and recruiters, so that job market mismatches can be reduced. The centrality measure of skills helps to develop farsighted programmes for the training of digital skills for job market entrants and targeted re-education campaigns.

\section{Approach and Preliminary Findings}

For the first exploratory part of the research, 5,227 job posting from Germany's most popular online job portal, stepstone.de, are considered: All job postings advertised with 
the tag "Artificial Intelligence' 3 are processed to a Document-Term-Matrix of Documents (y) and terms (x) is created. Here, terms in the form of unigrams (python), bigrams (machine learning), and trigrams (natural language processing) are considered. The recent work by Dawson et. al [5] provides a comprehensive list of relevant skills. Based on this matrix, weighted networks of skills are created from all postings. Skill tags are connected as nodes in a network, if they appear in the same advertisement. Figure 1 shows the joint occurrences of skills, measured as share of the number of all job postings.

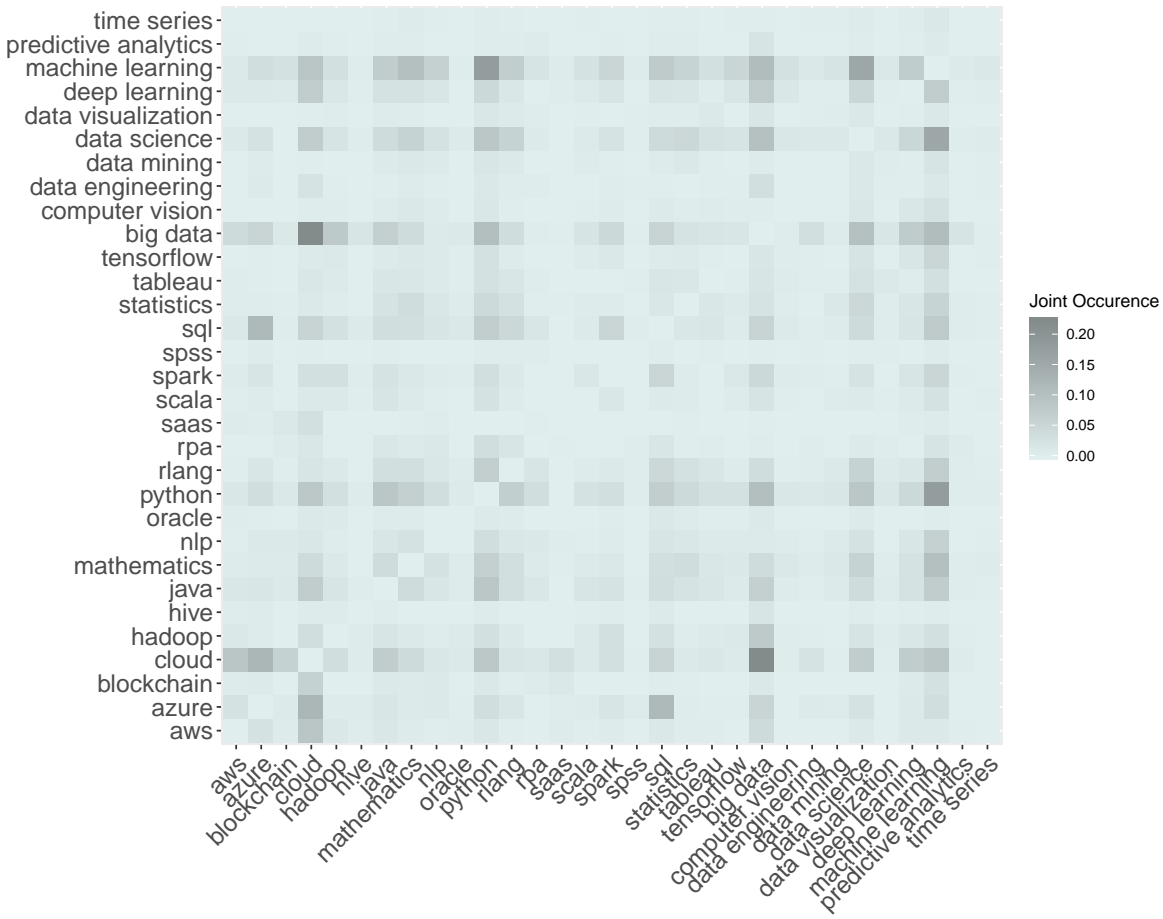

Fig. 1. The skill heatmap depicts the relative frequency of co-occurrences of certain skills, e.g., big data skills, such as cloud, azure, or aws (Amazon Web Services) are frequently (15 to 20 per cent of all postings) mentioned together.

Previous work in this field [6/7/8] suggests that regional ecosystems should have distinct patterns of demand for ICT skills. Therefore, this work examines regional skill networks separately. The given dataset allows to create skill networks for at least 36

\footnotetext{
${ }^{3}$ Collected between January 15th and February 28th 2020 with the use of web-parsing and scraping algorithms developed in Python.
} 
German urban areas (including major cities, such as Berlin, Hamburg, Munich, Frankfurt, and Cologne, but likewise key industrial areas, such as Dortmund, Essen, Stuttgart or Wolfsburg). Figure 2 illustrates two regional networks with the example of Frankfurt (am Main) and Hamburg.

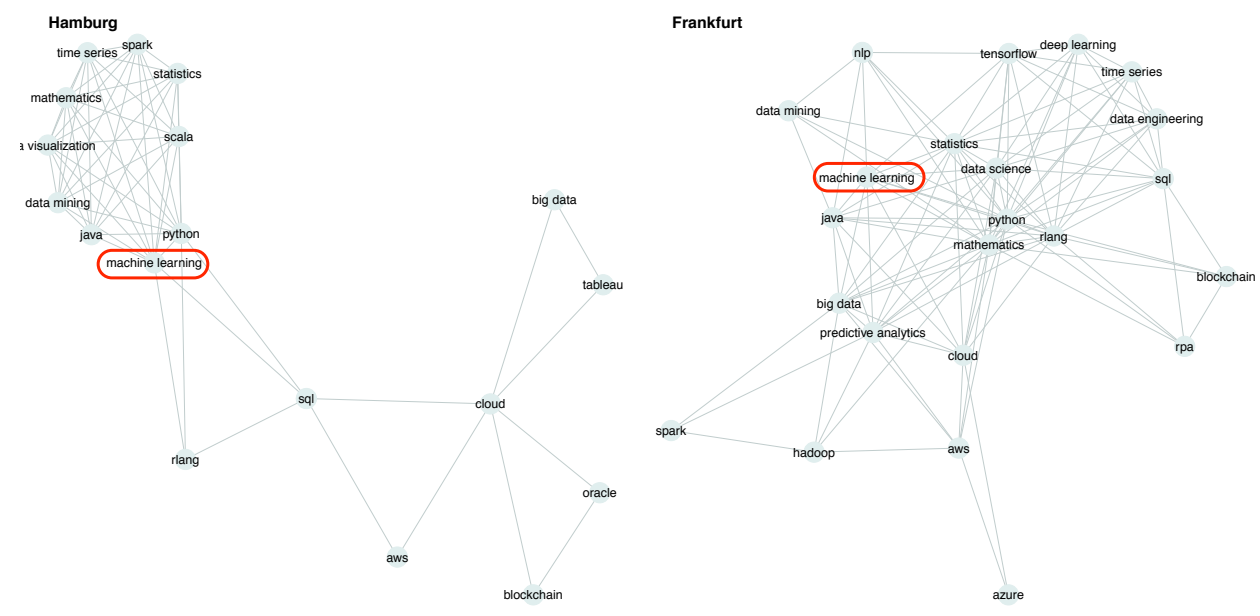

Fig. 2. Two skill networks are constructed with the example of job posts from Hamburg (lhs) and job advertisements from Frankfurt (am Main) (rhs). Two skills are connected if jobs have jointly advertised the same skills. While the Frankfurt network is closely connected, the Hamburg network is less densely connected and allows to recognise at least two distinct cliques at first sight. The Eigenvector centrality of skills that connect different network cliques, e.g. machine learning, hence is much higher in Hamburg (1.00) than in Frankfurt (0.29)

While the Frankfurt network is closely connected, the Hamburg network is less densely connected and allows to recognise at least two distinct cliques at first sight. For a comparative analysis of skills, which is independent of the network setting, the weighted Eigenvector centrality of each skill is calculated. This measure considers not only the centrality of each node, but also the centrality of its adjacent nodes. It could therefore be considered as a scale independent measure of skill importance in each ecosystem. In the given example, the Eigenvector centrality of the skill machine learning that connects different network cliques in only one network, hence is much higher in Hamburg (1.00) than in Frankfurt (0.29). 
Additional inferential statistical analysis suggests that differences in skill connectivity, measured by average betweenness centrality of the network, is driven by a) number of job postings, b) number of firms advertising, and c) heterogeneity of industry types. Further extensions of this work will focus on this aspect of analysis. Likewise, future extensions should identify cliques of specific skills by clustering algorithms. Lastly, extension might consider the development of skill tags over time.

Summary. With the use of online data from Germany's major job platform networks of digital skills are created around the topic of Artificial Intelligence. Initial research indicates there is a sizable difference in the importance of skill tags across German cities, depending on the industrial fingerprint of the region. The findings of this work could be used in order to create a data-driven strategy for the acquisition and the development of adequate skills needed to implement and leverage new technologies at best.

\section{References}

1. Yoo, Youngjin, Ola Henfridsson, and Kalle Lyytinen. "Research commentary-the new organizing logic of digital innovation: an agenda for information systems research.” Information systems research 21, no. 4 (2010): 724-735.

2. Nambisan, Satish, Kalle Lyytinen, Ann Majchrzak, and Michael Song. "Digital Innovation Management: Reinventing innovation management research in a digital world." Mis Quarterly 41, no. 1 (2017).

3. De Mauro, Andrea, Marco Greco, Michele Grimaldi, and Paavo Ritala. "Human resources for Big Data professions: A systematic classification of job roles and required skill sets." Information Processing \& Management 54, no. 5 (2018): 807-817.

4. Stephany, Fabian, and Hanno Lorenz. 2019. "Back to the Future - Changing Job Profiles in the Digital Age." SocArXiv. August 16. doi:10.31235/osf.io/9jyag.

5. Dawson, Nik, et al. "Adaptively selecting occupations to detect skill shortages from online job ads." arXiv preprint arXiv:1911.02302 (2019).

6. Stephany, Fabian, and Fabian Braesemann. "An exploration of wikipedia data as a measure of regional knowledge distribution." In International Conference on Social Informatics, pp. 31-40. Springer, Cham, 2017.

7. Stephany, Fabian, Fabian Braesemann, and Mark Graham. 2019. "Coding Together Coding Alone: The Role of Trust in Collaborative Programming." SocArXiv. May 3. doi:10.31235/osf.io/8rf2h.

8. Stephany, Fabian. 2019. "Everything You Always Wanted to Know About AI - Nowcasting Digital Skills with Wikipedia.” SocArXiv. September 18. doi:10.31235/osf.io/rfzdq. 
